\title{
Validation Study on Retinal Vessel Caliber Measurement Technique
}

Citation for published version (APA):

Huang, F., Dashtbozorg, B., Zhang, J., Yeung, A. K. S., Berendschot, T. T. J. M., \& ter Haar Romeny, B. M. (2018). Validation Study on Retinal Vessel Caliber Measurement Technique. In J. M. R. S. Tavares, \& R. M. Natal Jorge (Eds.), VipIMAGE 2017. ECCOMAS 2017 (pp. 818-826). Springer, Cham. Lecture Notes in Computational Vision and Biomechanics Vol. 27 https://doi.org/10.1007/978-3-319-68195-5_89

Document status and date:

Published: 01/01/2018

DOI:

10.1007/978-3-319-68195-5_89

Document Version:

Publisher's PDF, also known as Version of record

Document license:

Taverne

Please check the document version of this publication:

- A submitted manuscript is the version of the article upon submission and before peer-review. There can be important differences between the submitted version and the official published version of record.

People interested in the research are advised to contact the author for the final version of the publication, or visit the DOI to the publisher's website.

- The final author version and the galley proof are versions of the publication after peer review.

- The final published version features the final layout of the paper including the volume, issue and page numbers.

Link to publication

\footnotetext{
General rights rights.

- You may freely distribute the URL identifying the publication in the public portal. please follow below link for the End User Agreement:

www.umlib.nl/taverne-license

Take down policy

If you believe that this document breaches copyright please contact us at:

repository@maastrichtuniversity.nl

providing details and we will investigate your claim.
}

Copyright and moral rights for the publications made accessible in the public portal are retained by the authors and/or other copyright owners and it is a condition of accessing publications that users recognise and abide by the legal requirements associated with these

- Users may download and print one copy of any publication from the public portal for the purpose of private study or research.

- You may not further distribute the material or use it for any profit-making activity or commercial gain

If the publication is distributed under the terms of Article $25 \mathrm{fa}$ of the Dutch Copyright Act, indicated by the "Taverne" license above, 


\title{
Validation Study on Retinal Vessel Caliber Measurement Technique
}

\author{
Fan Huang ${ }^{1(凶)}$, Behdad Dashtbozorg ${ }^{1}$, Jiong Zhang $^{1}$, Alexander Yeung ${ }^{2}$, \\ Tos T.J.M. Berendschot ${ }^{2}$, and Bart M. ter Haar Romeny ${ }^{1,3}$ \\ 1 Department of Biomedical Engineering, Eindhoven University of Technology, \\ 5600MB Eindhoven, The Netherlands \\ \{F.Huang, B.Dasht. Bozorg, J .Zhang1, B.M.TerHaarRomeny\}@tue.nl \\ 2 University Eye Clinic Maastricht, Maastricht, The Netherlands \\ T.Berendschot@maastrichtuniversity.nl \\ 3 Department of Biomedical and Information Engineering, \\ Northeastern University, Shenyang 110000, China
}

\begin{abstract}
Changes in retinal vessel caliber are associated with several diseases, such as diabetes and hypertension. The robust assessment of abnormality on vessels with different sizes is a challenging task. In this paper, we propose a robust and reliable method for the measurement of retinal vessel caliber. The method is validated on a dataset where the optic disc centered images are acquired using 6 different fundus cameras with a repetitive acquisitions. The results are compared with the semiautomatic software IVAN, where the relative errors are similar.
\end{abstract}

Keywords: Vessel width $\cdot$ Arteriolar-to-venular ratio $\cdot$ Fundus images · Active contour · Diabetes

\section{Introduction}

In the past few years, retinal image analysis has been used for computer-aided diagnosis in large-scale screening programs. It utilizes automatic algorithms for the retinal landmarks detection including the vasculature and the optic disk, and biomarkers quantification such as the vessel caliber and the curvature $[1,4,5]$.

Change in retinal vessel caliber is an important indicator of vascular abnormality caused by diseases like diabetes and hypertension [7]. According to the literature, vessel width related biomarkers like the central retinal arteriolar equivalent (CRAE), the central retinal venular equivalent (CRVE) and the arteriolarto-venular ratio (AVR) [10] are significantly associated with the progress of diabetes, hypertension and other cardiovascular diseases $[8,9]$. However, recent clinical studies on retinal vascular caliber changes still rely on a semi-automatic software: IVAN developed by University of Wisconsin, USA more than 20 years ago $[3,11,12]$. According to our experience, assessing the vessel calibers of one 
retinal image, with resolution $3456 \times 2304$, using IVAN takes around 7-10 min, including automatic vessel detection and classification, manual cropping, adding vessel segments and correcting their labels. In large-scale screening programs, analysis of retinal images manually using IVAN software is time consuming, exhausting and prone to human error.

Therefore, developing an automatic software for vessel caliber measurement with comparable performance to the IVAN software is still an open challenge. In this paper, we present a vessel width assessment method based on an active contour technique combined with validation studies. We examine the proposed method by comparing its performance with IVAN on the same images. Additionally, we conduct an intra-camera variability test using images from 6 different fundus cameras to test the robustness of the method.

\section{Methodology}

The vessel caliber measurement requires an accurate vessel boundary segmentation. However, most automatic vessel segmentation techniques apply vessel enhancement on retinal images, and then rely on a global threshold value to obtain a binary vessel map. The choice of the threshold value strongly affects the quality of the segmentation, which may result in inaccurate vessel width estimations. The vessel width measurement presented in this paper uses the centerlines of segmented vessels as the initial control points for an active contour method. The contour is deformed iteratively to locate the left and right boundaries of vessels. Finally, the vessel caliber is measured by computing the distance from one detected vessel edge to the other one.

\section{(a) Vessel centerline extraction}

The proposed method is initialized by extracting the vascular tree. We used the vessel segmentation technique proposed by Zhang et al., which employs a set of multi-scale Gaussian derivative filters rotated to different directions in the socalled 'orientation-scores' [13]. This method provides an enhanced vessel map, which is then converted to a binary vascular map using a proper threshold value.

On the segmentation map, vessels within the optic disc region are eliminated by the optic disc mask obtained using the SEF filters proposed by Dashtbozorg et al. [6]. Then an iterative thinning algorithm is used to obtain the centerline of the vasculature. Junction points like vessel branchings and crossings are also removed, resulting in a map of several individual separated vessel segments. Afterwards, short segments are removed and the remained vessel segments are used in the next step for the caliber measurement.

\section{(b) Artery/vein classification}

In this work, we have used a supervised technique to classify the vessels into arteries and veins. Firstly, for each centerline pixel, we extract in total 455 features including: the local intensity of RGB and HSB color channels; the mean, standard deviation, median, minimum and maximum of the intensities inside small, medium and large circular regions; the intensity values along each vessel 
centerline; and the intensity inside each vessel segment. After that, a geneticsearch based feature selection approach is used to select the subset of features giving the highest classification accuracy from the 455 features. Finally, a logistic regression classifier and the set of selected features are used for the classification of arteries and veins.

\section{(c) Active contour model construction}

In this step, the geodesic active contour model proposed by Caselles et al. [2] for solving global optimization problem is exploited to locate the left and right edges for each segment. First of all, an enclosed and deformable contour $\mathbf{x}(t)=(x(t), y(t))(t \in(0,1))$ is initialized using the extracted centerline pixels. Afterwards, the surface is iteratively deformed to minimize an energy function:

$$
E(\mathbf{x})=\int_{0}^{1} E_{\text {int }}(\mathbf{x}(t))+E_{\text {ext }}(\mathbf{x}(t))+E_{\text {con }}(\mathbf{x}(t)) d t
$$

where $E_{\text {int }}$ is the force (internal energy) resulted by the interaction between adjacent control points, which preserves the smoothness of the surface. While $E_{\text {ext }}$ is the image gradient (external energy) which pulls the contour toward vessel boundary, and $E_{c o n}$ is a constraint for the external force. Therefore, at each iteration, the control points follow the contour evolution equation:

$$
\begin{gathered}
\frac{\partial \mathbf{x}(t))}{\partial t}=\alpha g(I)(c+\kappa) \vec{n}+\beta(\nabla g(I) \cdot \vec{n}) \vec{n}+\gamma \nabla \mathbf{x}(t) \cdot \nabla g(I), \\
g(I)=\frac{1}{1+\nabla I^{2}},
\end{gathered}
$$

where $I(x, y)$ is the image and $\nabla I$ is the first-order Gaussian derivative of $I, \kappa$ and $\vec{n}$ are the euclidean curvature and the unit normal vector of $\mathbf{x}(\mathrm{t}), g(I)$ is the speed function given $\nabla I$ and $c, \alpha, \beta$ and $\gamma$ are weighting parameters. The evolution is terminated when the stop criterion is satisfied, resulting in a smooth vessel edge detection.

\section{(d) Vessel caliber measurement}

The vessel caliber is estimated using the evolved contour (see Fig. 1). After the contour stops at the vessel boundary, it is split into two sections by removing the control points at the two ends of the vessel segments, forming the left and right vessel edges. Then for each control point on one side of the vessel, a corresponding nearest point is found on a B-spline interpolated curve of two nearest points on the other side. The Euclidean distance between each two points is computed. The distance is then converted to micrometer $\mu m$ using the pixel size of each image, which is estimated by the general optic disc diameter $1400 \mu \mathrm{m}$ which is used by the semi-automated retinal image analysis software (IVAN) and the diameter (with unit in pixel) measured by the method described in [6]. In order to prevent outliers, the measured distances with extreme values are eliminated and the vessel width is calculated as the average of the remained measurements. 


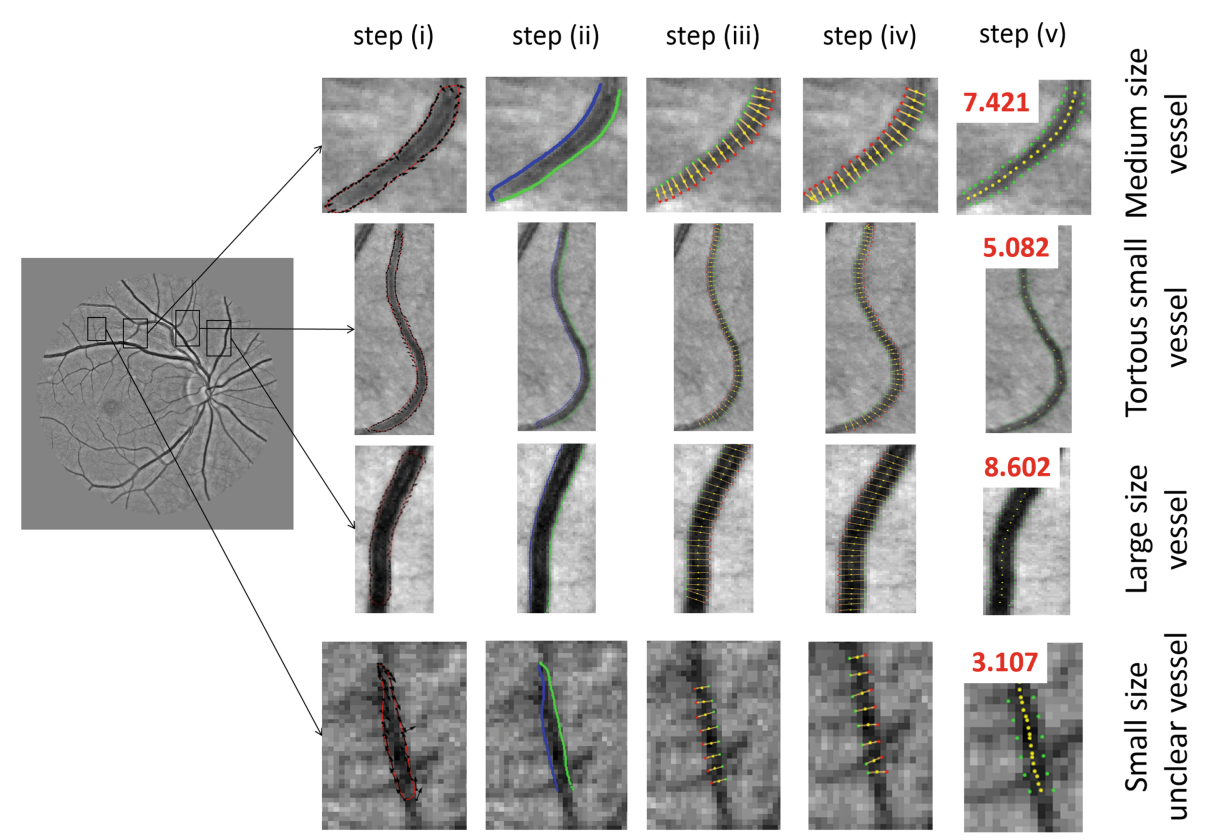

Fig. 1. The vessel caliber measurement is applied on multiple sizes of retinal vessels in 5 steps: (i) vessel boundary is detected by the active contour algorithm, (ii) the contour is split into two sections, (iii)-(iv) for each point on one side of the vessel, a nearest point is found on the other side, ( $\mathrm{v}$ ) vessel caliber is estimated as the average of the pixel distances between the two boundaries, and then converted to $\mu \mathrm{m}$ using the actual pixel size of each camera.

\section{Experimental Result}

\subsection{Material}

In order to validate the repeatability and reliability of the proposed method for vessel width measurement, we built up a dataset where the retinal images are obtained from 12 healthy subjects using 6 different fundus cameras, including (1) 3nethra classic, (2) Canon Cr-1 Mark II, (3) Topcon NW300, (4) Nidek AFC230, (5) EasyScan and (6) Spectralis HRA OCT. The first four cameras are color fundus camera and the last two cameras use a different imaging modality the Scanning Laser Ophthalmology (SLO) (see Fig. 2). Each subject repeatedly received acquisition 5 times by each camera on the right eye with optic-disc centered.

Retinal vessels on every image are detected by the introduced segmentation method, and the vessel centerlines are extracted. For each subject, the vessel segments are indexed and considered for the validation if they are well detected in more than 3 out of 5 acquisitions. The validation study includes examining the intra-camera variability and inter-camera variability in the proposed method. 


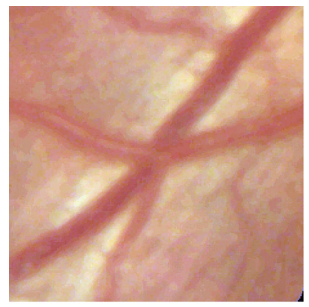

(a) 3nethra classic

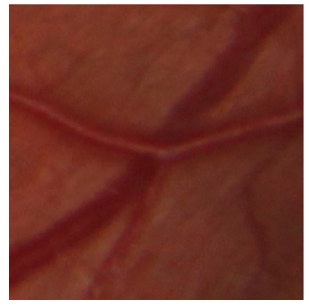

(d) Nidek AFC-230

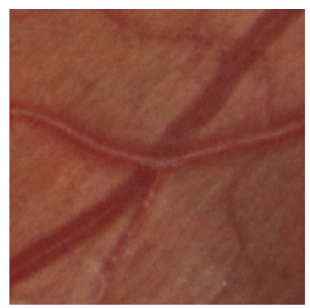

(b) Canon Cr-1 Mark II

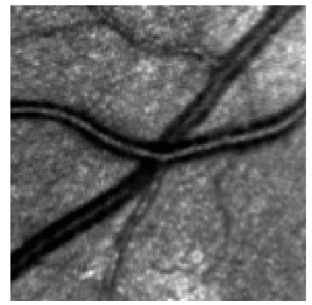

(e) EasyScan

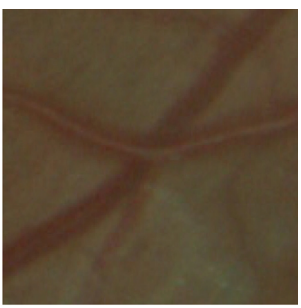

(c) Topcon NW300

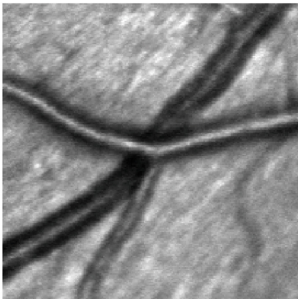

(f) Spectralis HRA OCT

Fig. 2. The image patches are acquired on the same subject by multiple fundus cameras, where the measured caliber values are used in an intra-camera and inter-camera variability studies.

The former study aims to validate the repeatability of the caliber value measured on the same vessel but acquired in different acquisitions per camera. The latter study aims to compare the caliber values estimated on the same vessel acquired by multiple cameras.

\subsection{Comparison with IVAN}

In this section, we compare our results with the IVAN software on the measurement of common biomarkers: CRAE, CRVE and AVR values using the images of the Canon camera. First of all, the vessels within the standard area of 0.5 to 1.0 disc diameters are selected and then classified into artery and vein. After the automatic classification, the wrongly classified vessels are manually corrected by the program developed by Dashtbozorg et al. [5]. The calibers of selected vessels are measured using the proposed active contour method. The CRAE, CRVE and AVR values are computed using the revised formulas proposed by Knudtson et al. [10]. In the case of IVAN, the same images are semi-automatically processed following its instruction. The comparisons in terms of CRAE, CRVE and AVR are shown in Fig. 4, where the dashed lines in the figures are the linear regression line indicating the agreement of two methods. As we can see, our automatic width measurement technique shows good alignment with the IVAN software which is commonly used in clinical studies. 

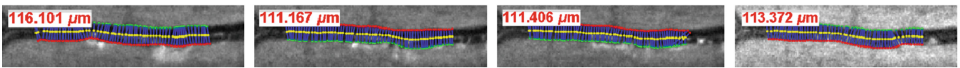

$112.01 \mathrm{~mm}$

(a) Caliber measured on large size vessel acquired in 5 acquisitions.
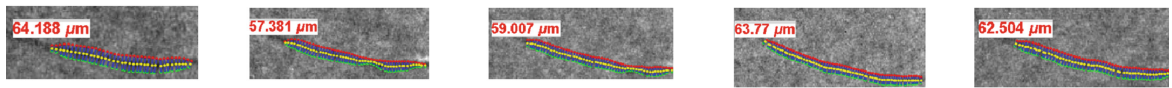

(b) Caliber measured on small size vessel acquired in 5 acquisitions.
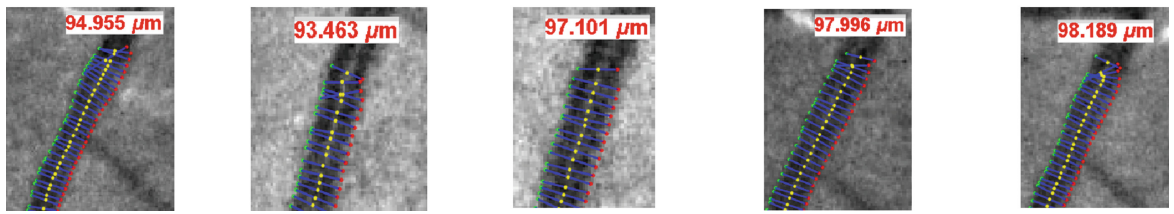

(c) Caliber measured on medium size vessel acquired in 5 acquisitions.

Fig. 3. Width measurement results with an intra-camera variability study on vessels with various sizes using the Canon Cr-1 Mask II camera. We obtain the estimated width for (a) large vessel: $112.81 \pm 2.03 \mu \mathrm{m}$ with a relative error of $1.7 \%$, (b) small vessel: $61.37 \pm 3.02 \mu \mathrm{m}$ with a relative error of $4.9 \%$, (c) medium vessel: $96.34 \pm 2.06 \mu \mathrm{m}$ with a relative error of $2.1 \%$.

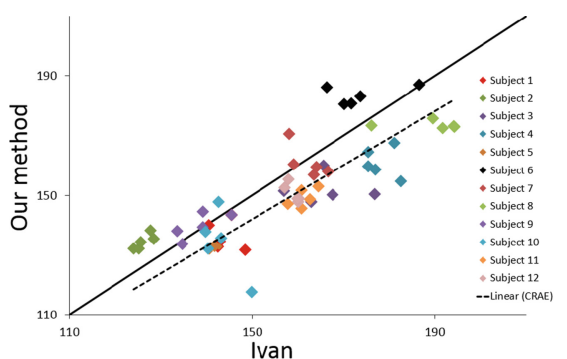

(a) CRAE

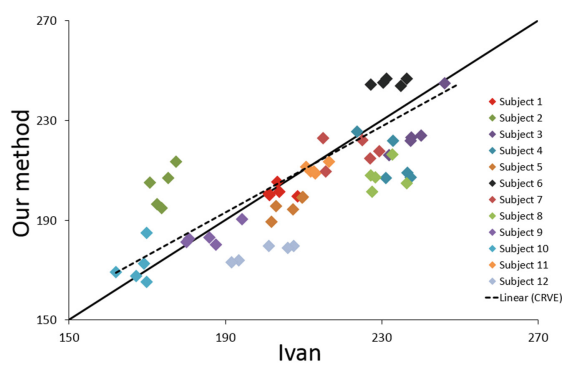

(b) CRVE

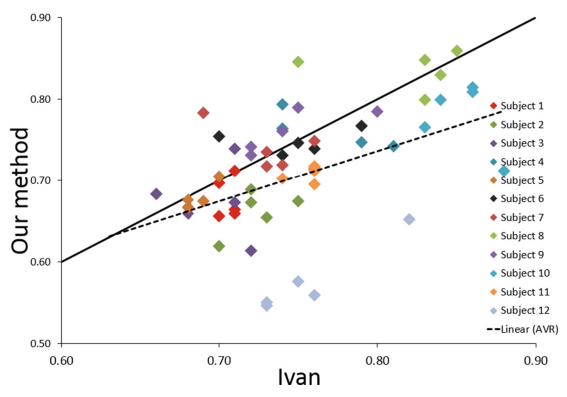

(c) AVR

Fig. 4. Scatter plots for comparing the (a) CRAE, (b) CRVE and (c) AVR obtained by the proposed method and the IVAN software on the retinal images acquired using Canon camera. The dashed line show the linear regression line for the data points. 


\subsection{Intra-camera Validation}

In this section, we validate the proposed method on the 5 images of the same subject, same camera, named intra-camera validation (see Figs. 3 and 5 as exam-

Table 1. Intra-camera validation: relative errors computed on the images of 3nethra, Canon, Nidek, Topcon, EasyScan and Spectralis by using the proposed method and the IVAN software.

\begin{tabular}{l|l|l|l|l|l|l|l}
\hline Method & Camera & $\begin{array}{l}\text { Pixel-size } \\
(\mu \mathrm{m} / \text { pixel })\end{array}$ & Resolution & \multicolumn{5}{|l}{ Relative error } \\
\hline & & & & CRAE & CRVE & AVR & Average \\
\hline IVAN software & Canon & 4.35 & $3456 \times 2304$ & $2.32 \%$ & $1.91 \%$ & $2.65 \%$ & $2.29 \%$ \\
\hline Our method & Canon & 4.35 & $3456 \times 2304$ & $2.84 \%$ & $2.40 \%$ & $3.20 \%$ & $3.52 \%$ \\
\hline & 3 nethra & 4.83 & $2048 \times 1536$ & $3.59 \%$ & $2.71 \%$ & $3.44 \%$ & $3.24 \%$ \\
\hline & Nidek & 2.73 & $3744 \times 3744$ & $3.91 \%$ & $5.00 \%$ & $6.33 \%$ & $5.08 \%$ \\
\hline & Topcon & 7.0 & $2048 \times 1536$ & $2.74 \%$ & $1.41 \%$ & $3.06 \%$ & $2.41 \%$ \\
\hline & EasyScan & 10.75 & $1024 \times 1024$ & $4.48 \%$ & $8.07 \%$ & $8.52 \%$ & $7.02 \%$ \\
\hline & Spectralis & 5.11 & $1536 \times 1536$ & $2.22 \%$ & $2.04 \%$ & $2.50 \%$ & $2.25 \%$ \\
\hline & & & & & & & \\
\hline & & & & & & \\
\hline & & & & & & &
\end{tabular}

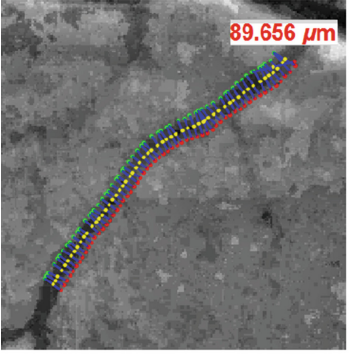

(a) 3nethra classic

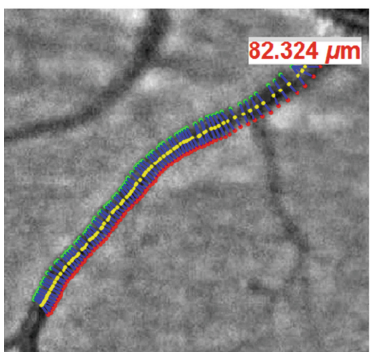

(d) Nidek AFC-230

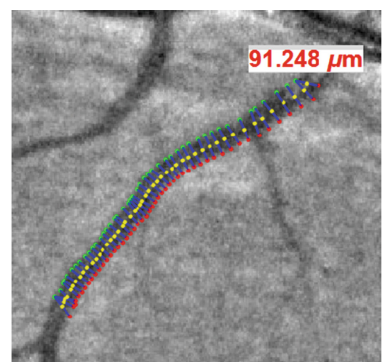

(b) Canon Cr-1 Mask II

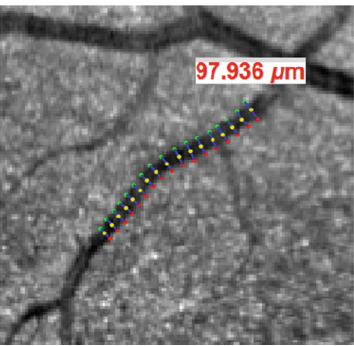

(e) EasyScan

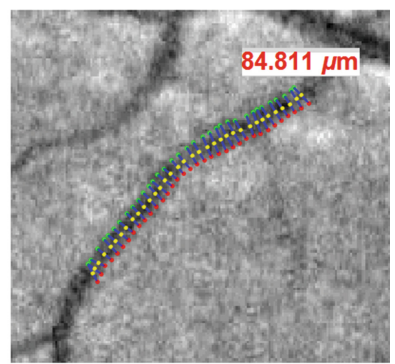

(c) Topcon NW300

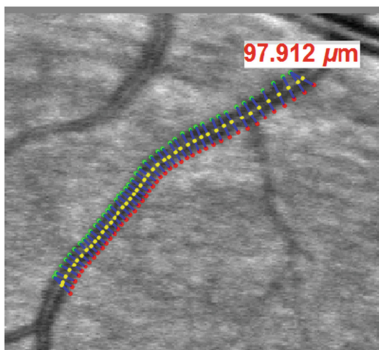

(f) Spectralis HRA OCT

Fig. 5. The vessel width measured with different imaging modalities. On the high resolution cameras (b) Canon Cr-1 mask II, (d) Nidek AFC-230 and (f) Spectralis HRA OCT, we obtain values with low relative error $2.8 \%$. The vessel width is overestimated on the low resolution cameras (a) 3nethra classic, (b) Topcon NW300 and (e) EasyScan. 
ples), by calculating the relative error of the measured CRAE, CRVE and AVR values. The biomarkers are computed using the pipeline described in Sect. 3.2. In addition, we compute the relative error of the biomarkers obtained by IVAN on images from Canon camera. The values obtained by IVAN are considered as the reference ones. The average relative errors for each camera are shown in Table 1.

As we can see from the table, the intra-camera variability of proposed method is comparable with the semi-automatic software. The average error on the Canon images is $2.29 \%$ using IVAN software, while our method has an average error of $3.5 \%$, which is slightly higher than the one obtained by IVAN. However, on the images of EasyScan, our method produces $7.02 \%$ error, which is much higher than the other cameras. It is because that the images of EasyScan are of low resolution $(10 \mu \mathrm{m} /$ pixel $)$, which means missing 1 pixel during width measurement making $10 \mu \mathrm{m}$ variation at the end. This implies that vessel width measurement on the images of a low resolution camera has high risk, thus these images are not suitable for quantitative analysis.

\section{Conclusion}

Measuring the caliber of vessels in different sizes is an open task in retinal image analysis. In this paper, we propose a method for vessel width estimation and we validate it on a dataset where the images were acquired on multiple subjects with different fundus cameras. The experimental results show that our method gives consistent width measurements for retinal images in different acquisition setups using various cameras.

Acknowledgements. This work is part of the Hé Programme of Innovation Cooperation, which is financed by the Netherlands Organization for Scientific Research (NWO), dossier No. 629.001.003.

\section{References}

1. Bekkers, E.J., Zhang, J., Duits, R., ter Haar Romeny, B.M.: Curvature based biomarkers for diabetic retinopathy via exponential curve fits in SE(2). In: Proceedings of the Ophthalmic Medical Image Analysis Second International Workshop, OMIA 2015, Held in Conjunction with MICCAI 2015, pp. 113-120. Iowa Research Online (2015)

2. Caselles, V., Kimmel, R., Sapiro, G.: Geodesic active contours. Int. J. Comput. Vision 22(1), 61-79 (1997)

3. Chew, M., Xie, J., Klein, R., Klein, B., Cotch, M.F., Redline, S., Wong, T.Y., Cheung, N.: Sleep apnea and retinal signs in cardiovascular disease: the MultiEthnic Study of Atherosclerosis. Sleep Breath. 20(1), 15-23 (2016)

4. Dashtbozorg, B., Abbasi-Sureshjani, S., Zhang, J., Huang, F., Bekkers, E., ter Haar Romeny, B.M.: Infrastructure for retinal image analysis. In: Proceedings of the Ophthalmic Medical Image Analysis Second International Workshop, OMIA 2016, Held in Conjunction with MICCAI 2016, Athens, Greece, 17th October 2015, pp. 105-112. Iowa Research Online (2016) 
5. Dashtbozorg, B., Zhang, J., Abbasi, S., Huang, F., ter Haar Romeny, B.M.: Retinal health information and notification system (RHINO). In: SPIE Medical Imaging, pp. 1013,437-1013,437-6. International Society for Optics and Photonics (2017)

6. Dashtbozorg, B., Zhang, J., Huang, F., ter Haar Romeny, B.M.: Automatic optic disc and fovea detection in retinal images using super-elliptical convergence index filters. Image Analysis and Recognition. Lecture Notes in Computer Science, vol. 9730, 697-706 (2016)

7. ter Haar Romeny, B.M., Bekkers, E.J., Zhang, J., Abbasi-Sureshjani, S., Huang, F., Duits, R., Dashtbozorg, B., Berendschot, T., Smit-Ockeloen, I., Eppenhof, K.A.J., Feng, J., Hannink, J., Schouten, J., Tong, M., Wu, H., van Triest, H.W., Zhu, S., Chen, D., He, W., Xu, L., Han, P., Kang, Y.: Brain-inspired algorithms for retinal image analysis. Mach. Vis. Appl. 27, 1-19 (2016)

8. Ikram, M.K., Janssen, J.A., Roos, A.M., Rietveld, I., Witteman, J.C., Breteler, M.M., Hofman, A., Van Duijn, C.M., de Jong, P.T.: Retinal vessel diameters and risk of impaired fasting glucose or diabetes. Diabetes 55(2), 506-510 (2006)

9. Kifley, A., Wang, J.J., Cugati, S., Wong, T.Y., Mitchell, P.: Retinal vascular caliber and the long-term risk of diabetes and impaired fasting glucose: the blue mountains eye study. Microcirculation 15(5), 373-377 (2008)

10. Knudtson, M.D., Lee, K.E., Hubbard, L.D., Wong, T.Y., Klein, R., Klein, B.E.: Revised formulas for summarizing retinal vessel diameters. Curr. Eye Res. 27(3), 143-149 (2003)

11. McEvoy, C., Wallace, I., Hamill, L., Neville, C., Hunter, S., Patterson, C., Woodside, J., Chakravarthy, U., Young, I., McKinley, M.: Increasing fruit and vegetable intake has no effect on retinal vessel caliber in adults at high risk of developing cardiovascular disease. Nutr. Metab. Cardiovasc. Dis. 26(4), 318-325 (2016)

12. Sarwar, S., Sadiq, M.A., Hanout, M., Nguyen, Q.D.: Assessment of retinal vessel caliber changes in eyes with non-neovascular age-related macular degeneration after progression to neovascular age-related macular degeneration. Graefe's Arch. Clin. Expe. Ophthalmol. 254(3), 599-601 (2016)

13. Zhang, J., Bekkers, E.J., Abbasi, S., Dashtbozorg, B., ter Haar Romeny, B.M.: Robust and fast vessel segmentation via Gaussian derivatives in orientation scores. In: International Conference on Image Analysis and Processing, pp. 537-547 (2015) 